\title{
Abordagem interprofissional à população em situação de rua: relato de experiência
}

\author{
Interdisciplinary approach to homeless street people: experience report
}

\author{
Larissa Oliveira Barbosaa, Tayná Coelho Nascimento ${ }^{a}$, Maria Alice Barbosa Fortunatob, \\ Marcelo Moreira Corgozinhoc \\ a Acadêmica de Enfermagem da Universidade Católica de Brasília. Brasília, DF, Brasil. \\ 'Enfermeira. Mestre em Ciências da Saúde pela Universidade de Brasília. Professora da Escola de Saúde e Medicina da \\ Universidade Católica de Brasília. Brasília, DF, Brasil. \\ cEnfermeiro. Doutor em Bioética pela Faculdade de Saúde da Universidade de Brasília. Professor da Escola de Saúde e Medicina da \\ Universidade Católica de Brasília. Brasília, DF, Brasil.
}

RESUMO Objetivo: Relatar a experiência vivenciada por duas acadêmicas do curso de enfermagem da Universidade Católica de Brasília, após acompanhar uma equipe de consultório na rua.

Descrição da Experiência: A experiência foi vivenciada junto à equipe de uma Região Administrativa do Distrito Federal, onde foram realizadas atividades de redução de agravos voltadas à população em situação de rua. Trata-se de uma população que merece atenção especial em virtude da sua marginalização, estigmatização e invisibilidade social. A equipe interprofissional desempenha importante função social na redução de agravos, pois a interdisciplinaridade promove a troca de saberes que qualificam o produto final das ações em saúde.

Conclusão: Considera-se que esta vivência junto ao consultório na rua contribuiu para a formação profissional das acadêmicas de enfermagem, pautada no desenvolvimento de posicionamento crítico e reflexivo quanto à responsabilidade social dos profissionais e gestores frente às populações específicas em extrema vulnerabilidade social.

Palavras-chave: sistema único de saúde; atenção primária à saúde; equipe interdisciplinar de saúde; população de rua.

Objective: To report the experience of two nursing undergraduate students from Universidade Católica de Brasília, after following a team who brings health care to street people.

Description of the experience: The case was experienced with the staff of an Administrative Region of the Federal District, where activities were carried out to mitigate health injuries to street people. These people deserve special attention due to marginalization, stigmatization, and social invisibility. The interdisciplinary team plays an important social role in the reduction of diseases, promoting the exchange of knowledge that qualifies the final product of health actions.

Conclusion: According to the students, their experience with street people contributed to their professional training as nursing students. It played a key role in their critical and reflexive thinking regarding the social responsibility of professionals and managers towards specific populations submitted to extreme social vulnerability.

Keywords: Brazilian health care system; primary health care; interdisciplinary health team; street people.

\section{Correspondência:}

MARCELO MOREIRA CORGOZINHO

Universidade Católica de Brasília - Secretaria da Escola de Saúde

QS 7, LT 01 - Águas Claras

71966-700 Brasília, DF, Brasil

E-mail: mmcorgozinho@gmail.com 


\section{INTRODUÇÃO}

O consultório na rua surgiu em Salvador, Bahia, em meados dos anos 1990, como resposta à problemática em que se encontravam crianças e adolescentes em condições de rua e uso de drogas ${ }^{1}$. Os principais motivos que se destacam e induzem os indivíduos a se estabelecer nas ruas são sequencialmente: problemas de alcoolismo e outras drogas, desemprego e rompimento de vínculo familiar. Em determinadas situações tais motivos apresentam correlação entre si ou um ser consequência do outro².

Os consultórios na rua são dispositivos públicos integrantes da rede de atenção integral em saúde mental, que proporcionam ações de promoção, prevenção e cuidados primários diretamente no espaço da rua ${ }^{3}$. A mudança do consultório "de" rua para consultório "na" rua não caracteriza apenas uma modificação de nomenclatura, pois essa alteração está associada com a transformação na Politica Nacional de Atenção de Saúde Mental, que representa um olhar diferenciado para a população em situação de rua. Hodiernamente, existem mais de cem consultórios na rua em todo o território brasileiro, fornecendo prática clínica de cuidado que percorre a rede de cuidados ao propiciar a inserção desse grupo específico aos serviços de saúde. Devido à necessidade de várias demandas distintas, o consultório é um serviço transversal que oferece desde a atenção em saúde mental, até as práticas de atenção básica em saúde

Ao considerar os princípios estabelecidos pelo Sistema Único de Saúde (SUS) que versam sobre o acesso aos serviços de saúde, foi criada, no ano de 2009, a Política Nacional para a População em Situação de Rua ${ }^{4}$, enquanto que a Portaria no 122 de 2011 definiu as diretrizes para organização e funcionamento das equipes de consultório na rua (ECR) ${ }^{2-4}$. Assim, a atenção básica deve analisar cada ser humano dentro da sua singularidade, ou seja, no aspecto individual e coletivo, sendo a porta de entrada prioritária dos usuários com o sistema de saúde 2 .

A exposição a diversos fatores relacionados aos riscos vividos pelos moradores de rua revela o porquê da necessidade de ter sido criada uma política direcionada a eles ${ }^{3}$. Os riscos comumente encontrados são aqueles vinculados às violências, ao uso de álcool e outras drogas, precariedade de qualidade de vida, exclusão social, em especial a cobertura limitada pelas equipes de saúde da família (ESF) ${ }^{3}$. Além disso, esse público alvo enfrenta barreiras burocráticas no acesso à saúde, por exemplo, a solicitação de documentos como o Cartão do SUS, comprovante de residência e identificação pessoal ${ }^{5}$.

Dessa forma, o consultório na rua atua como elo entre o morador em situação de rua e o serviço de saúde, sendo uma ferramenta itinerante de saúde que unifica a rede de atenção básica e promove atividades de atenção psicossocial ${ }^{5-6}$. A ECR é constituída de equipe interprofissional composta por médico; enfermeiro; psicólogo; assistente social; terapeuta ocupacional; técnico em enfermagem e técnico em higiene bucal - dentre as atividades da equipe incluem a busca ativa de pacientes e o cuidado aos usuários de substâncias psicoativas. Para isso, os profissionais desempenham suas atividades de forma integrada com as Unidades Básicas de Saúde (UBS), equipes dos Centros de Atenção Psicossocial (CAPS), serviços de urgência e emergência, dentre outros ${ }^{7}$.

Os preceitos da assistência integral estão intimamente associados à interdisciplinaridade e representa a eficiente integração entre os diferentes campos de conhecimentos; enquanto a interprofissionalidade consiste na intensa interação entre diferentes profissionais ${ }^{8}$. Ressalta-se que é de suma importância que os membros da equipe estejam dispostos a criar vínculos de confiança e acolhimento com essa população ${ }^{3-4}$. Como crítica, a literatura descreve que a maioria das equipes tem feito apenas o cadastramento e o encaminhamento dos usuários para as unidades de saúde mais próximas, o que não é o ideal, afinal a maioria da população em situação de rua está em uma situação de total vulnerabilidade e não se deslocará até aos locais indicados pela equipe ${ }^{9}$.

A população em situação de rua é heterogênea, no entanto, tem em comum a fragilidade que o viver na rua propicia $^{7}$. A realidade a qual é referida está submersa na circunstância de desigualdade social expressa pelas precárias condições de vida, além do fardo pesado da invisibilidade como ser humano ${ }^{10}$.

Contudo, advoga-se pela estimulação de estudos e reflexões que tratem dos desafios da instituição de linhas de cuidado que atendam às necessidades impostas pela vida na rua, e que viabilizem a criação de vínculo entre os profissionais de saúde e os indivíduos estigmatizados socialmente ${ }^{5}$. Diante do exposto, este estudo objetiva relatar a experiência vivenciada por duas acadêmicas do curso de enfermagem da Universidade Católica de Brasília, após acompanhar uma ECR.

\section{DESCRIÇÃO DA EXPERIÊNCIA}

Trata-se de um relato de caráter descritivo sobre as experiências vivenciadas por duas acadêmicas de enfermagem, a partir do acompanhamento da ECR de uma Região Administrativa do Distrito Federal-DF. As atividades foram desenvolvidas durante o internato de saúde comunitária, que tem carga horária de 30 horas semanais 4 meses de duração. As participações junto à ECR aconte- 
ceram nas tardes de quinta-feira, sob a orientação dos preceptores e enfermeiro da equipe.

A ECR era composta pelo médico, enfermeiro, psicólogo, assistente social, técnico de enfermagem, agente de saúde pública e os acadêmicos de enfermagem. No primeiro momento, foi disponibilizado material teórico para que as acadêmicas se aproximassem com a temática abordada. Depois, houve a participação junto à reunião de equipe, para o planejamento das atividades de modo que cada paciente fosse visto de forma individualizada. Esse debate relacionado às estratégias a serem empregadas ocorre anteriormente por uma reunião conhecida como "pré-campo" e, posteriormente, é realizada também uma reunião "pós-campo", para avaliar a eficácia das atividades implementadas pelo consultório na rua.

Inicia-se a organização dos recursos materiais necessários para atender a cada demanda estabelecida na reunião de "pré-campo". Ocorre, então, a separação dos medicamentos básicos; materiais para curativos; preservativos; insumos para testes rápidos de doenças infectocontagiosas, materiais para as coletas de exames; imunobiológicos; material de apoio didático para palestras; e formulários para prescrição, solicitação de exames e encaminhamentos aos serviços de apoio.

A abordagem na rua era condicionada à liberação do transporte disponibilizado pela Regional de Saúde, onde a equipe realizava períodos de visita na semana. Os atendimentos aos pacientes ocorriam em diversos locais em que os mesmos "residiam na rua", como nas proximidades de padarias, supermercados, restaurantes, feiras permanentes, abrigos, albergues, reciclagens, dentre outros. Vale ressaltar que a ECR acolhe tanto a população que mora literalmente na rua, como aquela população que tira o seu sustento das ruas, como: Guardadores de carro, vendedores ambulantes e até mesmo a população que reside em condições precárias de moradia.

O percurso da visita do consultório é planejado nas reuniões da equipe, no entanto, pode ser mudado a qualquer momento ou necessidade, chegando a atender nas adjacências de lixões, rodoviárias, hospitais, CAPS e demais localidades, ou seja, no local onde estiverem os pacientes em situação de rua. Esse acolhimento acontece também para os pacientes que são encaminhamentos por outras unidades de saúde, pessoas encaminhadas por membros da sociedade e outros órgãos públicos.

O perfil dos pacientes atendidos é variado, mas foi observado que a maioria era dependente químico, principalmente usuários de álcool, maconha e crack. É salientada pela ECR a necessidade de capacitação específica para a abordagem junto à população em situação de rua, para que o profissional compreenda a psicodinâmica da vida nas ruas, a identificação de situações de perigo e vulnerabilidade. Dessa forma, o acolhimento acontece em conjunto com todos os profissionais da equipe, onde são analisadas as necessidades individuais de cada indivíduo. Observou-se que a equipe promovia a escuta qualificada para atender as demandas imediatas dos pacientes e, em diversas situações, faziam a mediação ou ponte com outros serviços de saúde como: CAPS, ESF e Hospitais Regionais do DF.

Assim, ao analisar a prática da ECR é possível caracterizála como uma valiosa estratégia de interação entre serviços de saúde e a comunidade, a partir da redução de danos em saúde.

\section{DISCUSSÃO}

O principal motivo que induz os indivíduos a se estabelecer nas ruas é a utilização de drogas ${ }^{2}$, sendo que a maioria da população em situação de rua é composta por indivíduos do sexo masculino ${ }^{4}$. No que tange ao sexo feminino, a situação é agravada pela vulnerabilidade relacionada às condições de gênero, como a violência sexual e a consequente infecção sexualmente transmissível e gravidez indesejada - gravidez de risco ${ }^{4}$.

O acesso dos profissionais de saúde à população de rua é complexo devido à baixa adesão aos tratamentos propostos e ao acompanhamento irregular. A vida nas ruas possui uma psicodinâmica que começa com os fatores que levaram o indivíduo a ir para as ruas, indo até às adaptações de sua vivência diária, como as formas para garantir a sobrevivência em meio à hostilidade do ambiente ${ }^{3}$.

A ECR é constituída por uma equipe interprofissional, que busca o olhar diferenciado e livre do sentimento de piedade ou salvação, que foca apenas nas necessidades de cada indivíduo e na sua singularidade - favorecendo a criação de vínculos com a equipe ${ }^{3}$. Para isso, o acolhimento e escuta qualificada tornam-se tecnologias primordiais. $\mathrm{O}$ acolhimento aparece na área das tecnologias leves, relacionado às relações que se estabelecem entre trabalhadores e usuários, nos modos de escutas, nas maneiras de lidar com as situações imprevistas, nas formas de sensibilidade do trabalhador e no posicionamento ético situacional ${ }^{11}$.

O acolhimento dos usuários exige reflexão por parte da equipe quanto ao conjunto de ofertas disponibilizadas para lidar com as necessidades de saúde da população. As ofertas devem estar à disposição para serem consideradas na realização da escuta qualificada da demanda, ou seja, é importante que seja estabelecida a forma como os diferentes profissionais participarão do acolhimento: Quem vai receber ou abordar inicialmente o usuário do serviço, como avaliar 
ou classificar a vulnerabilidade, a ação imediata dispensada, e quando encaminhar/agendar uma consulta ${ }^{11}$.

É fundamental que ocorra a fase "pré-campo" para realizar o mapeamento e a consequente priorização dos problemas de acordo com a respectiva demanda da região, tendo como prioridade a análise dos locais onde apontam o maior índice de gravidade ${ }^{5}$. Nesse momento é verificada a disponibilidade de recursos materiais e transporte, pois a insuficiência de veículos é um desafio que a equipe enfrenta para se deslocar até os usuários em situação de rua ${ }^{12}$.

A participação das acadêmicas de enfermagem foi baseada na observação direta dos atendimentos dispensados pelos profissionais da ECR, junto à população de rua. Observou-se que não existe protocolo estabelecido para a ordem da abordagem interdisciplinar-interprofissional, no entanto, a equipe iniciava com a abordagem mediada pelo psicólogo, seguido pelo médico, enfermeiro e, por último, o assistente social. A primeira abordagem interprofissional ocorreu pela mediação do psicólogo, com uma breve conversa sobre a situação de rua, alimentação, presença de vícios, fontes de renda, condições de moradia na rua e violência. Assim, a dificuldade de acesso a locais para higiene pessoal possibilita a disseminação de doenças e dificulta a prevenção e a promoção de saúde. Quanto aos hábitos de higiene, os principais locais utilizados para tomar banho e executar necessidades fisiológicas são os albergues, a própria rua e os banheiros públicos ${ }^{10}$. Constantemente, essa população se depara com situações de violência que muitas vezes pode acarretar a própria morte e, mesmo sim, é nas ruas que buscam por acolhimento ${ }^{13}$.

O médico examinava os pacientes, ouvindo suas queixas e avaliando a presença de lesões e/ou sinais flogísticos. Pode ser prescrito medicações de rotina, e para aqueles pacientes que necessitam de medicação de uso contínuo é essencial o acompanhamento regular pela ECR, ESF ou CAPS. A etinerância dos locais de moradia torna-se uma barreira para o estabelecimento de vínculo com a equipe e o acesso à medicação de uso contínuo. Através do desejo e necessidade do paciente são ofertados insumos que objetivam a redução de agravos em saúde, como: preservativos, seringas, vacinas, kit de higiene bucal, entre outros.

O enfermeiro realizava a consulta de enfermagem que inclui parte da escuta inicial. A técnica de enfermagem oferecia os testes rápidos para detecção de HIV, sífilis, hepatites B e C, porém alguns pacientes os recusam. O enfermeiro realizava a avaliação e a classificação das úlceras, os curativos necessários, além de orientar a forma de cuidados das lesões. Observou-se que o enfermeiro exercia o papel de educador e facilitador e não somente assistencial e/ou gerencial. Além disso, os profissionais devem ter o olhar integral do indivíduo e desenvolver ações que proporcionem saúde, por meio de escuta qualificada e fortalecimento de vínculos ${ }^{6}$.

Como a maioria da população em situação de rua utiliza álcool e drogas ilícitas ${ }^{13}$, na superioridade das vezes o assistente social realizava as orientações acerca do CAPS e sua abrangência, e a técnica de enfermagem questionava sobre os comparecimentos às consultas, quando agendadas previamente. Destaca-se que o aumento ininterrupto do consumo de drogas é um dos maiores problemas de saúde pública no mundo ${ }^{13}$.

O Centro de Atenção Psicossocial Álcool e Drogas (CAPSad) atua no planejamento e implantação de copiosas estratégias que visam diminuir os danos e riscos motivados pelo abuso e dependência de substâncias psicoativas, o que possibilita o fortalecimento de elementos que propiciam a proteção da saúde e a inserção do indivíduo na comunidade. Dispõe de atendimento diário que possibilita o planejamento terapêutico inserido em uma visão individualizada que propicia formas de intervenção precoce, substanciando o estigma vinculado ao tratamento ${ }^{14}$.

A estigmatização refere-se às crenças negativas amplamente marcadas que excluem determinado indivíduos ou um grupo social especifico. Essas crenças geram como consequência uma atitude de indiferença que reforçam o processo de legitimação da invisibilidade social, ou seja, sabe-se que eles existem, mas é como não tivessem um rosto, uma identidade e uma história de vida. A condição de exclusão social é reflexo da forma como a sociedade reconhece tais pessoas: reconhecimento marcado pelos estigmas da marginalidade, da criminalidade e da miséria ${ }^{3}$.

Outra estratégia de apoio social é o Centro de Referência de Assistência Social (CRAS), uma unidade socioassistencial de proteção social básica, que tem como principal alvo a prevenção da ocorrência de danos em situações que envolvem a fragilidade social. Tem como fundamento a capacidade do engrandecimento de vínculos familiares, extrafamiliares e o acesso aos direitos de cidadania. Desta maneira, os serviços comunitários são baseados na rede de cuidados proposta, que desencadeia uma série de atividades que se iniciam desde o atendimento individualizado medicamentos, terapias, orientação - até acolhimento em grupos ou oficinas terapêuticas ${ }^{14}$.

Para atingir um nível de saúde satisfatório as pessoas precisam atender suas necessidades humanas básicas, devem ser capazes de adotar a modificações de comportamentos, além de possuir dos meios essenciais à operacionalização de tais mudanças. A educação em saúde vem cooperar para que essas pessoas adquiram autonomia para detectar os meios para melhorar a sua vida ${ }^{10}$. 
As possibilidades futuras para que o indivíduo deixe a condição de rua consiste na manutenção do vínculo do paciente com a equipe interprofissional, sendo que o respectivo grau de vulnerabilidade será considerado. No momento em que o paciente solicita ajuda para deixar o ambiente de rua, o assistente social aciona os diversos recursos e redes de apoio, a saber: CAPSad, CRAS, Centros de Recuperação Filantrópicos, Conselho Tutelar, Santas Casas de Misericórdia e outras parcerias. É essencial a vontade do paciente em sair da rua, ou seja, nenhuma medida será tomada sem o seu consentimento.

Considera-se que esta vivência junto à ECR contribuiu para a formação profissional das acadêmicas de enfermagem, pautada na necessidade de posicionamento crítico e reflexivo quanto à responsabilidade social dos profissionais e gestores frente às populações específicas em vulnerabilidade social. A população em situação de rua merece atenção diferenciada em virtude da sua marginalização e invisibilidade social.

A equipe interprofissional desempenha importante função social na redução de agravos. Contudo, a dinâmica do consultório na rua consiste na compreensão do desafio de ter conhecimento e se apropriar de uma realidade para transformá-la ${ }^{10}$.

\section{REFERÊNCIAS}

1. Londero MFP, Ceccim RB, Bilibio LFS. Consultório de/na rua: desafio para um cuidado em verso na saúde. Interface Comun Saúde Educ. 2014;18:251-60. https://doi.org/10.1590/1807-57622013.0738

2. Brasil. Ministério da Saúde. Portaria n 122, de 25 de janeiro de 2011. Define as diretrizes de organização e funcionamento das equipes de consultório na rua [Internet]. 2012 [capturado 2018 Jun 07]. Disponível em: http://bvsms.saude.gov.br/bvs/saudelegis/ gm/2012/prt0122_25_01_2012.html
3. Nery Filho A, Valério ALR, Monteiro LF. Guia do projeto consultório de rua. Salvador: Cetad; 2012.

4. Brasil. Ministério da Saúde. Secretaria de Atenção à Saúde. Manual sobre o cuidado à saúde junto à população em situação de rua. Brasília: Ministério da Saúde; 2012.

5. Hallais JAS, Barros NF. Consultório na rua: visibilidades, invisibilidades e hipervisibilidade. Cad Saúde Pública. 2015;31:1497-504. https:// doi.org/10.1590/0102-311X00143114

6. Schervinski AC, Merry CN, Evangelista IC, Pachedo VC. Atenção à saúde da população em situação de rua. Extensio. 2017;14(26): 55-64.

7. Costa TS. Consultórios na rua do Distrito Federal e perfil dos profissionais de saúde no ano de 2015 [Monografia]. Brasília: Graduação em Saúde Coletiva da Universidade de Brasília; 2015.

8. Barr H. Interprofessional education: the genesis of a global movement. London: Centre for Advancement of Interprofessional Education; 2015.

9. Reis Junior AG. Estudo de caso da equipe de saúde da família para a população em situação de rua de Belo Horizonte-MG. Rio de Janeiro: ENSP/Fiocruz; 2011.

10. Menezes ACSA, Campos TD, Pinheiro SMA. Promoção de ações educativas de saúde na população em situação de rua. In: Ev'Ângela DS, Barros BR, organizadores. Extensão PUC Minas: Sujeitos, espaços e tempo de ação. Belo Horizonte: PUC; 2017.

11. Brasil. Ministério da Saúde. Departamento de Atenção Básica. Acolhimento à demanda espontânea. Brasília: Ministério da Saúde; 2013.

12. Sousa AR, Oliveira APP, Farias LT, Colares MC, Feitoza AR. Consultório de rua: nova forma de atenção em saúde. Tendên Enferm Profis. 2015;7(3):1640-45.

13. Silva FP, Frazão IS, Linhares FMP. Práticas de saúde das equipes dos consultórios de rua. Cad Saúde Pública. 2014;30:805-14. https:// doi.org/10.1590/0102-311X00100513

14. Silveira RWM, Rezende D, Moura WA. Pesquisa-intervenção em um CAPSad - Centro de Atenção Psicossocial Álcool e Drogas. Gerais (Univ. Fed. Juiz Fora). 2010;3(2):184-97. 